\title{
PO3-5-20 Poster session
}

\section{The Research Progress of Clinical Applications of Human Menstrual Blood- Derived Mesenchymal Stem Cells}

\author{
Yunke Huang, Yu Kang
}

\section{The Obstetrics \& Gynecology Hospital of Fudan University, China}

Mesenchymal stem cells (MSCs) are multipotent adult stem cells (ASCs) derived from mesoderm. It has the potential to differentiate into multiple tissues and organs. MSCs feature low tumorigenicity, more stable genome, less ethical limits than embryonic stem cells (EMCs), bringing fresh ideas to the clinical treatment of many diseases and has become a research hotspot in recent years.

MenSCs were found as early as 2007 by Cui to demonstrate the ability to differentiate into myocardial cells, and are as equally capable of differentiation as the other MSCs. It has been testified to differentiate into all three types of embryonal systems. MenSCs could be isolated and retrieved from healthy adult women's menstrual blood: density gradient centrifugation could be adopted to isolate and culture MenSCs. About 48h after cell passage, MenSCs enter log phase, and expressed CD29 strong positive, CD44 positive, CD34, CD45 and HLA - DR negative. MenSCs going through adipogenic and osteogenic induction after cell passage could proliferate to the number required by clinical treatment within a relatively short period of time and with no occurrence of genetic abnormality, a proof that they are blessed with advantages like rapid proliferation, stable karyotype and multpotency.

Researches on MenSCs have been mature in its separation and cultivation techniques, differentiation potential and characteristics, and won't be repeated in this article. As scientists began to explore more possible clinical applications of MenSCs, their prospects have been testified in an ever increasing number of disease cases. The transplant of MenSCs and its differentiated products as the most common practice has been proved effective to treat lung injury and cancer, acute liver injury, liver fibrosis, peripheral nerve injury and neuroglioma. In the treatment of gynecological diseases, MenSCs transplant offers a novel and effective therapy for premature ovarian failure and intrauterine adhesion helpless by normal drug and surgeries. In recent years, targeted therapy using adenovirus infected MenSCs as vehicle gene is gaining wide attention. In its treatment of neurogliocytoma and gynecological oncology, its capacity to get round human immune barrier and precisely target oncology cells is worthy of further exploration. 